\title{
Stimulation of the soluble guanylate cyclase (sGC) inhibits fibrosis by blocking non-canonical TGF $\beta$ signalling
}

\author{
Christian Beyer, ${ }^{1}$ Christoph Zenzmaier, ${ }^{2,3}$ Katrin Palumbo-Zerr, ${ }^{1}$ Rossella Mancuso, ${ }^{1}$ \\ Alfiya Distler, ${ }^{1}$ Clara Dees, ${ }^{1}$ Pawel Zerr, ${ }^{1}$ Jingang Huang, ${ }^{1}$ Christiane Maier, ${ }^{1}$ \\ Milena L Pachowsky, ${ }^{4}$ Andreas Friebe, ${ }^{5}$ Peter Sandner, ${ }^{6,7}$ Oliver Distler, ${ }^{8}$ \\ Georg Schett, ${ }^{1}$ Peter Berger, ${ }^{2}$ Jörg H W Distler ${ }^{1}$
}

\begin{abstract}
Handling editor Tore K Kvien
- Additional material is published online only. To view please visit the journal online (http://dx.doi.org/10.1136/ annrheumdis-2013-204508).

For numbered affiliations see end of article
\end{abstract}

\section{Correspondence to} Dr Jörg Distler, Department of Medicine 3 and Institute for Clinical Immunology, University of Erlangen-Nuremberg, Ulmenweg 18, Erlangen D-91054, Germany; Joerg.distler@uk-erlangen.de

Received 22 August 2013 Revised 31 January 2014 Accepted 2 February 2014 Published Online First 24 February 2014
CrossMark

To cite: Beyer $C$, Zenzmaier $C$, PalumboZerr $\mathrm{K}$, et al. Ann Rheum Dis 2015;74:1408-1416.

\section{ABSTRACT}

Objectives We have previously described the antifibrotic role of the soluble guanylate cyclase (sGC). The mode of action, however, remained elusive. In the present study, we describe a novel link between sGC signalling and transforming growth factor $\beta$ (TGF $\beta$ ) signalling that mediates the antifibrotic effects of the $s G C$.

Methods Human fibroblasts and murine sGC knockout fibroblasts were treated with the $\mathrm{SGC}$ stimulator BAY 41-2272 or the stable cyclic guanosine monophosphate (cGMP) analogue 8-Bromo-cGMP and stimulated with TGF $\beta$. sGC knockout fibroblasts were isolated from $\mathrm{sGCl}^{\mathrm{fl} / \mathrm{fl}}$ mice, and recombination was induced by Creadenovirus. In vivo, we studied the antifibrotic effects of BAY 41-2272 in mice overexpressing a constitutively active TGF- $\beta 1$ receptor.

Results $\mathrm{SGC}$ stimulation inhibited TGF $\beta$-dependent fibroblast activation and collagen release. sGC knockout fibroblasts confirmed that the $S G C$ is essential for the antifibrotic effects of BAY 41-2272. Furthermore, 8-Bromo-cGMP reduced TGF $\beta$-dependent collagen release. While nuclear $\mathrm{p}$-SMAD2 and 3 levels, SMAD reporter activity and transcription of classical TGF $\beta$ target genes remained unchanged, SGC stimulation blocked the phosphorylation of ERK. In vivo, sGC stimulation inhibited TGF $\beta$-driven dermal fibrosis but did not change p-SMAD2 and 3 levels and TGF $\beta$ target gene expression, confirming that non-canonical TGF $\beta$ pathways mediate the antifibrotic SGC activity.

Conclusions We elucidated the antifibrotic mode of action of the SGC that increases cGMP levels, blocks non-canonical TGF $\beta$ signalling and inhibits experimental fibrosis. Since SGC stimulators have shown excellent efficacy and tolerability in phase 3 clinical trials for pulmonary arterial hypertension, they may be further developed for the simultaneous treatment of fibrosis and vascular disease in systemic sclerosis.

\section{INTRODUCTION}

During fibrosis, excessive accumulation of extracellular matrix results in chronic scaring of affected organs. Fibrosis disrupts the physiological tissue homeostasis and causes organ failure. In systemic sclerosis (SSc), a prototypical fibrotic disease, fibrosis affects various organ systems, including the lungs, the heart and the gastrointestinal tract. ${ }^{1} 2$
Because of the severe impact on organ function, patients with SSc suffer from high morbidity and mortality. Effective antifibrotic therapies are urgently needed but not yet available in clinical routine. $^{3-5}$

Fibroblasts are key players in fibrosis. While the exact pathomechanisms remain unclear, inflammation, vascular disease and other external stimuli lead to a pathological activation of fibroblasts. The activated fibroblasts express contractile proteins and release excessive amounts of extracellular matrix components, in particular collagens, which accumulate in fibrotic tissue. Although the external stimuli may subside during the course of the disease, the pathological fibroblast activation persists. During later stages of fibrotic disease, paracrine and autocrine loops as well as epigenetic signatures maintain the pathological fibroblast activation. ${ }^{1} \quad{ }^{2}{ }^{6-9}$ Of note, patients with SSc often present during these late disease stages, when fibrosis has already become manifest. In these patients, therapies that specifically target fibroblast activation are considered most promising. ${ }^{3} 4$

The soluble guanylate cyclase (sGC) has a wellestablished role in regulating vascular tone and remodelling. Nitric oxide (NO) is the physiological ligand of the sGC. Clinical application of $\mathrm{NO}$ or NO-donating drugs, however, is hampered by insufficient biometabolism, rapid development of tolerance and non-specific interactions of NO with other biological molecules. In particular, prolonged application or high concentrations of NO induce oxidative stress, cause DNA damage, prompt lipid peroxidation and alter protein function, which includes oxidation of the sGC rendering the enzyme unresponsive to NO. ${ }^{10}{ }^{11}$ Since oxidative stress has been implicated in fibroblast activation, the toxic effects of NO may be particularly harmful in patients with SSc. ${ }^{1}$ In contrast to NO-donating drugs, sGC stimulators cause an NO-independent, direct stimulation of the $\mathrm{sGC}$ to overcome these limitations. $^{10}$

The sGC stimulator riociguat has shown excellent efficacy and tolerability in phase 3 clinical trials for the treatment of chronic thromboembolic pulmonary hypertension (CTPH) and pulmonary arterial hypertension (PAH), a frequent disease manifestation in SSc. ${ }^{12}{ }^{13}$ Moreover, we have 
demonstrated that sGC stimulation is effective and well tolerated in different inflammation-driven and inflammationindependent preclinical fibrosis models of SSc. ${ }^{14}$ Other groups have reported antifibrotic effects of sGC stimulation in experimental models of various tissues, ${ }^{15}$ including liver ${ }^{16}$ and kidney fibrosis, ${ }^{17} 18$ suggesting a general antifibrotic effect. The molecular mechanisms, however, by which sGC stimulation inhibits fibroblast activation and reduces experimental fibrosis have remained elusive.

In the present study, we demonstrate that sGC stimulation interferes with transforming growth factor $\beta$ (TGF $\beta$ ) signalling, one of the key pathways of fibrosis. Pharmacological and genetic approaches show that sGC activity increases cyclic guanosine monophosphate (cGMP) levels, inhibits SMAD-independent TGF $\beta$ signalling and reduces fibroblast activation and collagen release.

\section{MATERIALS AND METHODS \\ Fibroblast cultures}

Isolation and culture of human and murine fibroblasts were performed as described previously. ${ }^{14}{ }^{19-21}$ Human fibroblasts were obtained from skin biopsies of 7 patients with SSc and 10 healthy individuals. All patients with SSc presented with diffusecutaneous SSc, and biopsies were taken from lesional skin at the volar side of the forearm. All patients with SSc and healthy volunteers provided written informed consent as approved by the Institutional Ethics Committee.

Murine fibroblasts were isolated from skin of conditional $\mathrm{sGC}^{\mathrm{f} / \mathrm{fl}}$ mice, in which the exon 10 of the $\beta 1$ subunit of the $\mathrm{sGC}$ is embraced by loxP sites. Since the $\beta 1$ subunit is an essential component of both sGC isoforms (sGC1 and 2), genetic recombination by Cre activity led to a complete loss of sGC activity. $^{22} 23$ To induce Cre-mediated recombination, Ad-Cre-GFP viruses were used. The same adenoviral vector encoding for LacZ (Ad-LacZ) served as control (Vector Biolabs, Philadelphia, Pennsylvania, USA). Both viruses were applied with a multiplicity of infection (MOI) of 80 in Dulbecco's Modified Eagle Medium (DMEM) containing 0.5\% fetal bovine serum (FBS) for $24 \mathrm{~h}$, and medium was replaced after the infection period. Genetic recombination was confirmed by genotyping PCR using the Qiagen DNeasy Blood\&Tissue kit (Qiagen, Hilden, Germany) for DNA isolation.

\section{In vitro fibroblast experiments}

Fibroblasts from passages 4-8 were used for the experiments. Prior to experiments, human and murine dermal fibroblasts were cultured in DMEM containing 0.1\% FBS for up to $48 \mathrm{~h}$. Two hours after adding BAY 41-2272 or 8-Bromo-cGMP, fibroblasts were stimulated with recombinant TGF- $\beta 1(10 \mathrm{ng} / \mathrm{mL}$; R\&D Systems, Abingdon, UK) for 48, 72 or $144 \mathrm{~h}$ as indicated. Thereafter, supernatants were collected to measure collagen and insulin-like growth factor binding protein 3 (IGFBP3) content, and cells were lysed for RNA and protein analysis. BAY 41-2272 was kindly provided by Bayer Health Care (Wuppertal, Germany), and 8-Bromo-cGMP was purchased from Sigma-Aldrich (Taufkirchen, Germany). Both compounds were dissolved in dimethyl sulfoxide (DMSO) to stock concentrations of 10.0 and $5.6 \mathrm{mM}$. The final concentration of DMSO in all experiments did not exceed $0.1 \%$.

\section{Quantitative real-time PCR (qPCR)}

Total RNA isolation, reverse transcription and real-time PCR were performed as described previously and are detailed in the online supplement. ${ }^{19} 2024$

\section{Collagen measurements}

Total soluble collagen in cell culture supernatants was quantified by Sircol collagen assay according to the manufacturer's instructions (Biocolor, Belfast, UK).

\section{ELISA for IGFBP3}

IGFBP3 in cell culture supernatants was assessed by ELISA as recommended by the manufacturer (Mediagnost, Reutlingen, Germany).

\section{Western blot analysis}

Proteins were separated by SDS-PAGE and transferred to a polyvinylidene difluoride membrane. The membrane was incubated with the appropriate primary antibody and HRP-conjugated secondary antibodies (Dako, Glostrup, Denmark; Jackson ImmunoResearch Europe, Newmarket, UK). Blots were visualised by enhanced chemiluminescence. $\beta$-Actin and $\alpha$-tubulin antibodies were used as loading controls (Santa Cruz Biotechnology, Heidelberg, Germany). Antibodies against $\alpha$-smooth muscle actin ( $\alpha$-SMA) (Sigma-Aldrich), calponin-1 (Sigma-Aldrich), phosphorylated SMAD3 (Santa Cruz), phosphorylated Akt (eBioscience, San Diego, California, USA), phosphorylated c-Jun N-terminal kinase (JNK) (Abcam, Cambridge, UK) and phosphorylated and nonphosphorylated ERK (Cell Signaling, Boston, Massachusetts, USA) were used as primary antibodies. ${ }^{25}$

\section{Luciferase reporter assay}

Fibroblasts were transfected with a CAGA luciferase reporter construct using a Nucleofector Kit for Human Dermal Fibroblasts (Lonza, San Diego, California, USA). A common $\beta$-galactosidase reporter vector (Sigma-Aldrich) was used as control. Twenty-four hours after stimulation with TGF $\beta$, luciferase activity was determined using a microplate luminometer (Berthold Technologies, Bad Herrenalb, Germany) and a MRX ELISA reader (Dynex Technologies, Chantilly, USA).

\section{Immunofluorescence staining for phosphorylated SMAD2/3}

Formalin-fixed, paraffin-embedded sections from murine skin were stained with antibodies against p-SMAD2/3 (Santa Cruz Biotechnology, Heidelberg, Germany) and anti-Alexa Fluor 594-tagged secondary antibody (Invitrogen, Carlsbad, California, USA). ${ }^{26-28}$ Respective isotype controls were used to exclude unspecific staining. Fluorescence stainings were analysed using a Nikon Eclipse 80i microscope and quantified by Image J software V.1.46.

\section{Quantification of murine skin}

Murine skin was analysed as described previously ${ }^{7} \quad 14 \quad 19-21 \quad 24$ 29-32 and are detailed in the online supplement.

\section{Statistical analysis}

All data are presented as median with IQR. Differences between the groups were tested for their statistical significance by MannWhitney U non-parametric test unless otherwise indicated. $p$ Values are expressed as follows: $0.05>p>0.01$ as $* ; 0.01>p>0.001$ as $* * ; \mathrm{p}<0.001$ as $* * *$ p Values less than 0.05 were considered significant.

\section{RESULTS}

\section{sGC stimulation inhibits TGF $\beta$-dependent collagen release}

We have recently demonstrated that sGC stimulation is effective in inhibiting fibrosis in various in vitro and in vivo model systems of SSc. To further elucidate the antifibrotic effects of 


\section{Basic and translational research}

sGC stimulation, we incubated dermal fibroblasts with the sGC stimulator BAY 41-2272 and TGFß. While healthy and SSc dermal fibroblasts demonstrated only low sGC expression levels as assessed by the common $\beta 1$ subunit (see online supplementary figure S1A), we observed that BAY 41-2272 significantly reduced the stimulatory effects of TGF $\beta$ on COL1A1 and COL1A2 pro-collagen mRNA levels in both healthy and SSc dermal fibroblasts (figure 1A-D). Reduced pro-collagen mRNA levels by sGC stimulation resulted in decreased collagen release from healthy and SSc dermal fibroblasts (see online supplementary figure S1B,C). BAY 41-2272 did not change levels of collagen mRNA or protein in unstimulated fibroblasts (figure $1 \mathrm{~A}-\mathrm{D}$ and online supplementary figure S1B,C), suggesting that sGC stimulation has no or only minor effects on resting fibroblasts.

\section{sGC stimulation prevents and reverses TGF $\beta$-dependent fibroblast activation}

TGF $\beta$ stimulates resting fibroblasts to undergo myofibroblast differentiation with increased expression of contractile proteins such as $\alpha$-SMA and enhanced expression of calponin- 1 and IGFBP3. ${ }^{33} 34$ To investigate whether sGC stimulators can inhibit TGF $\beta$-induced myofibroblast differentiation, we co-incubated fibroblasts with TGF $\beta$ and BAY 41-2272. In a first set of experiments, BAY 41-2272 was added throughout the whole incubation period. In this setting, BAY 41-2272 completely prevented the stimulatory effects of TGF $\beta$ on $\alpha$-SMA (ACTA), calponin-1 (CNN1) and IGFBP3 (IGFBP3) mRNA and protein levels (figure 2A-E). In an additional set of experiments, we initiated BAY 41-2272 treatment after TGF $\beta$ had already induced
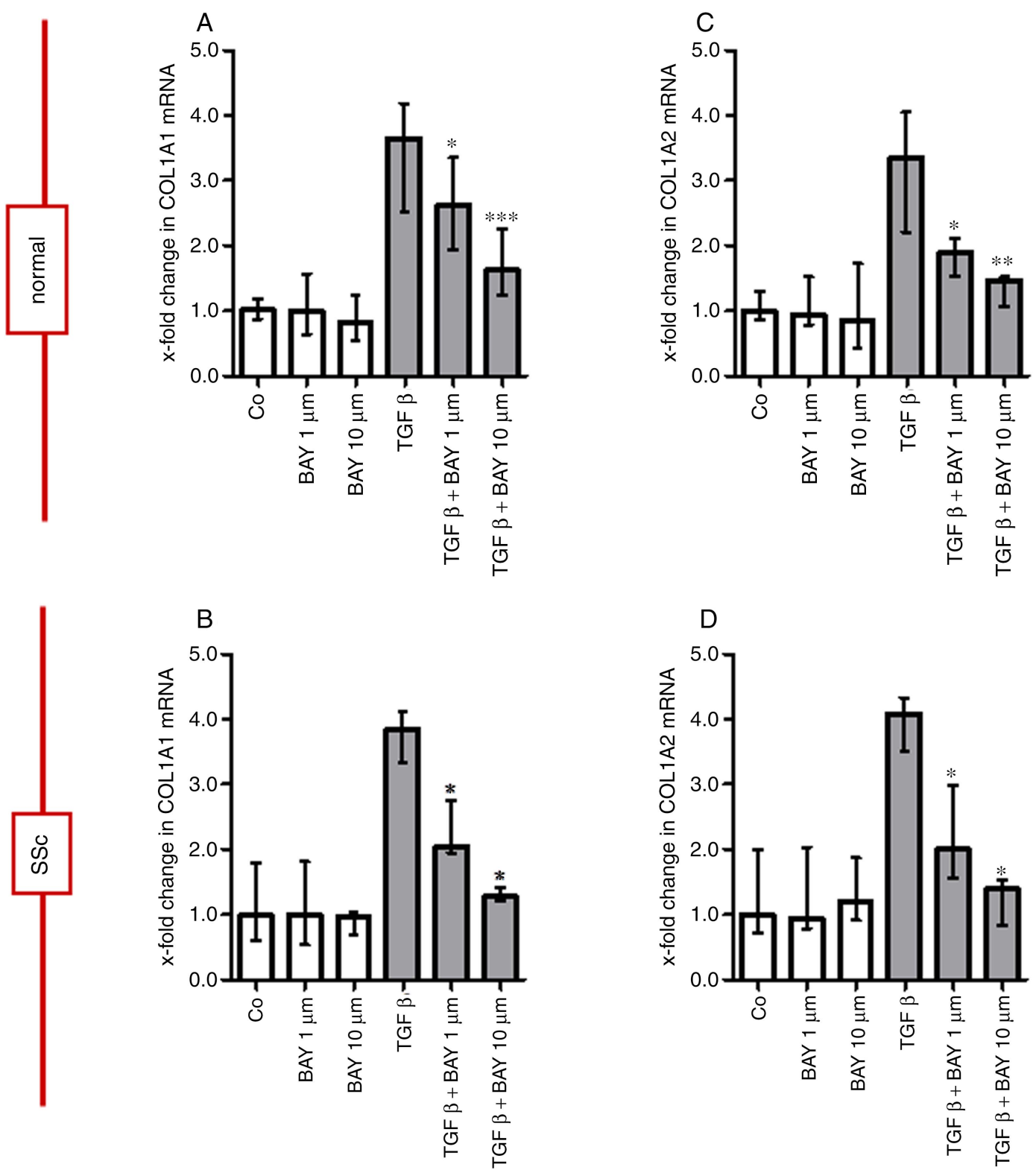

Figure 1 Treatment with the soluble guanylate cyclase stimulator BAY 41-2272 reduces transforming growth factor $\beta$ (TGF $\beta$ )-dependent COL1A1 and COL1A2 expression in fibroblasts isolated from healthy individuals and systemic sclerosis patients. (A-D) BAY 41-2272 was applied in doses of $1.0 \mu \mathrm{M}$ and $10.0 \mu \mathrm{M} 2 \mathrm{~h}$ before stimulation with $10 \mathrm{ng} / \mathrm{mL}$ TGF $\beta$ for $48 \mathrm{~h}$. All experiments are expressed as $x$-fold of the untreated and unstimulated control. $\mathrm{N} \geq 6$ for all experiments. (A, B) Pro-collagen COL1A1 mRNA levels with $\beta$-actin as internal controls. (C, D) Pro-collagen COL1A2 mRNA levels with $\beta$-actin as internal control. 

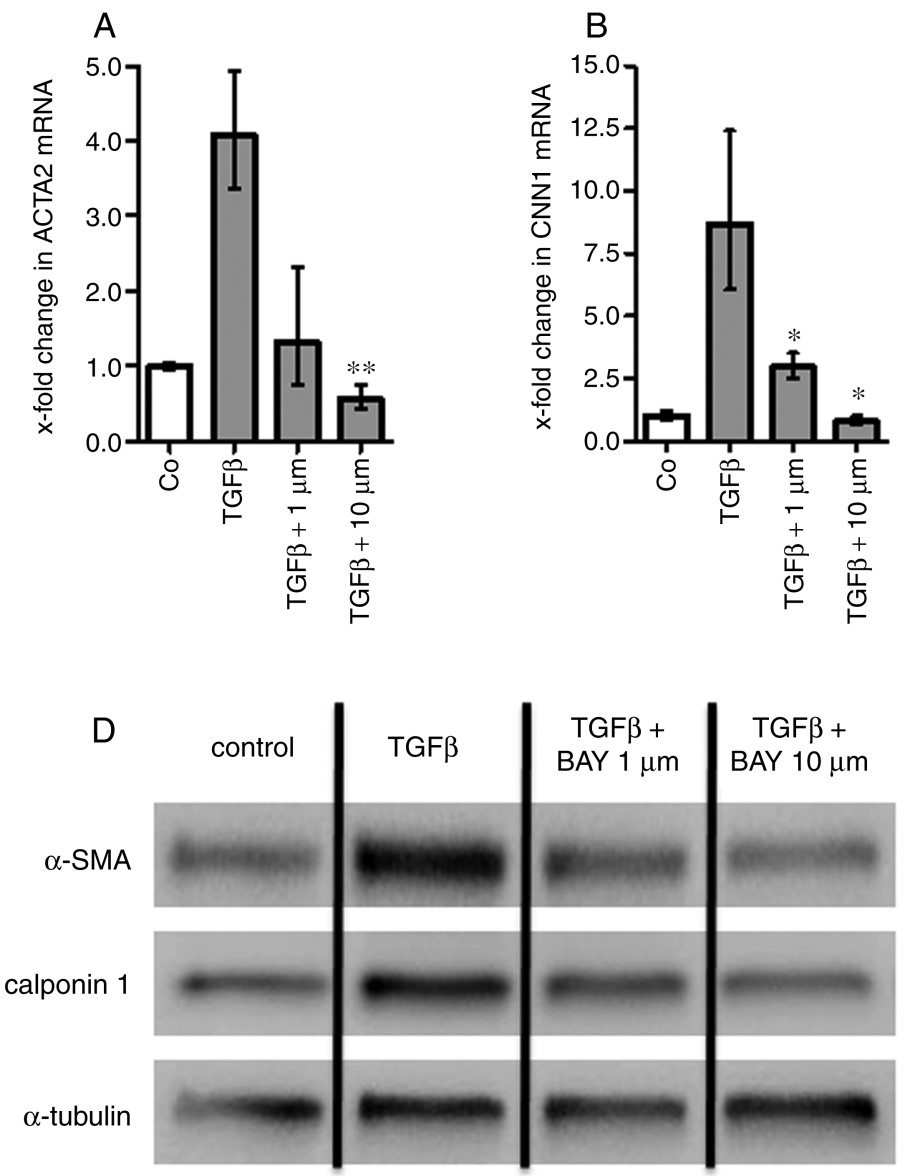
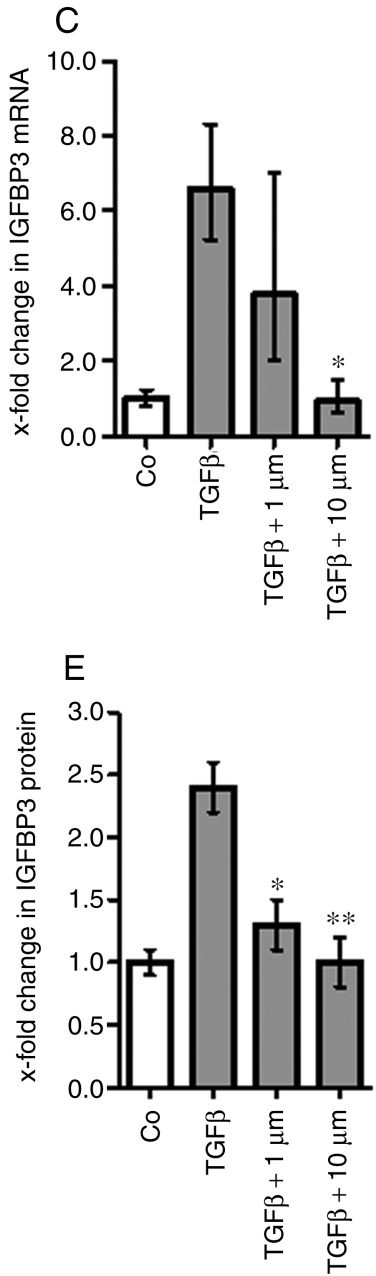

Figure 2 Soluble guanylate cyclase stimulation with BAY 41-2272 inhibits transforming growth factor $\beta$ (TGF $\beta$ )-induced fibroblast-to-myofibroblast differentiation. (A-E) BAY 41-2272 was applied in doses of $1.0 \mu \mathrm{M}$ and $10.0 \mu \mathrm{M}$ before stimulation with TGF $\beta$. Fibroblasts were stimulated with $10 \mathrm{ng} / \mathrm{mL}$ TGF $\beta$ for $72 \mathrm{~h}$. All experiments are expressed as $x$-fold of the unstimulated control. $\mathrm{N}=3$ for all experiments. A paired, two-sided $t$ test was used for statistical analysis. (A-C) ACTA2 ( $\alpha$-smooth muscle actin ( $\alpha$-SMA)), CNN1 (calponin-1) and IGFBP3 mRNA levels compared with HMBS as internal control. (D) Western blot analysis for $\alpha$-SMA and calponin-1. $\alpha$-Tubulin served as loading control. Representative images of three independent experiments using cells from different donors are shown. (E) Secreted IGFBP3 protein levels as determined by ELISA.

myofibroblast differentiation. In this setting, BAY 41-2272 reduced mRNA and protein levels for $\alpha$-SMA, calponin-1 and IGFBP3 almost back to baseline levels of resting fibroblasts (figure 3A-E). These findings suggest that sGC stimulation is effective in both preventing and reversing TGF $\beta$-dependent fibroblast activation.

\section{The antifibrotic effects of BAY 41-2272 are mediated via the sGC-cGMP axis}

We next confirmed the antifibrotic effects of the sGC-cGMP axis by an independent experimental approach and excluded off-targets effects of the sGC stimulator BAY 41-2272. We therefore analysed the effects of BAY 41-2272 on fibroblasts deficient for the sGC. To obtain sGC-deficient fibroblasts, we isolated fibroblasts from $\mathrm{sGC}^{\mathrm{f} / \mathrm{fl}}$ mice and induced genetic recombination with an Ad-Cre-GFP virus, while controls were treated with an Ad-LacZ virus. Loss of sGC activity did not change basal collagen release or collagen release upon TGF $\beta$ stimulation as assessed by COL1A1 and COL1A2 mRNA levels as well as collagen protein. While BAY 41-2272 effectively reduced the levels of col1a1 and col1a2 mRNA and collagen protein in fibroblasts with intact sGC activity (Ad-LacZ-treated controls), sGC stimulation did not inhibit the pro-fibrotic TGF $\beta$ effects in sGC knockout fibroblasts (Ad-Cre-GFP treated) (figure 4A-C). Thus, the antifibrotic effects of BAY 41-2272 depend on the $\mathrm{sGC}$ and are not mediated by off-target effects.

cGMP is the major downstream mediator of the sGC, but it is readily degraded in physiological states. To confirm the antifibrotic effects of sGC-cGMP signalling on an additional level, we used the stable cGMP analogue 8-Bromo-cGMP. 8-Bromo-cGMP is refractory to enzymatic degradation and thus mimics high cGMP levels by increased sGC activity. Similar to the sGC stimulator BAY 41-2272, 8-Bromo-cGMP inhibited TGF $\beta$-dependent collagen release as assessed by col1a1 and col1a2 mRNA and collagen protein (figure 4D-F).

\section{The antifibrotic activity of sGC-cGMP signalling is independent from canonical TGF $\beta / S M A D$ signalling and modifies ERK signalling}

We hypothesised that sGC-cGMP signalling mediates its antifibrotic effects by interfering with canonical TGF $/$ SMAD cascades. In canonical TGF $/$ SMAD signalling, the expression of specific target genes is induced by SMAD transcription complexes. TGF $\beta$ receptors phosphorylate SMAD2 and 3 proteins 

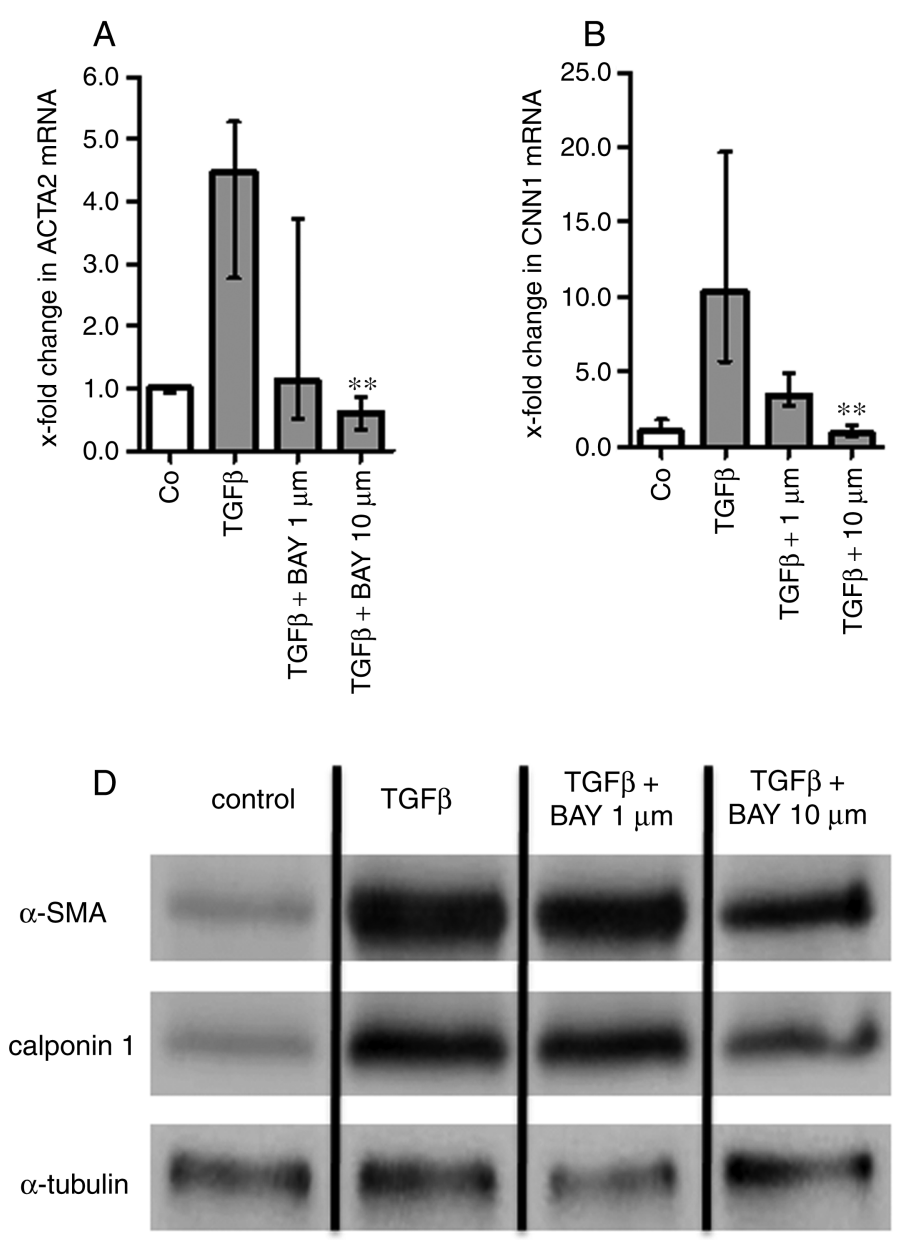
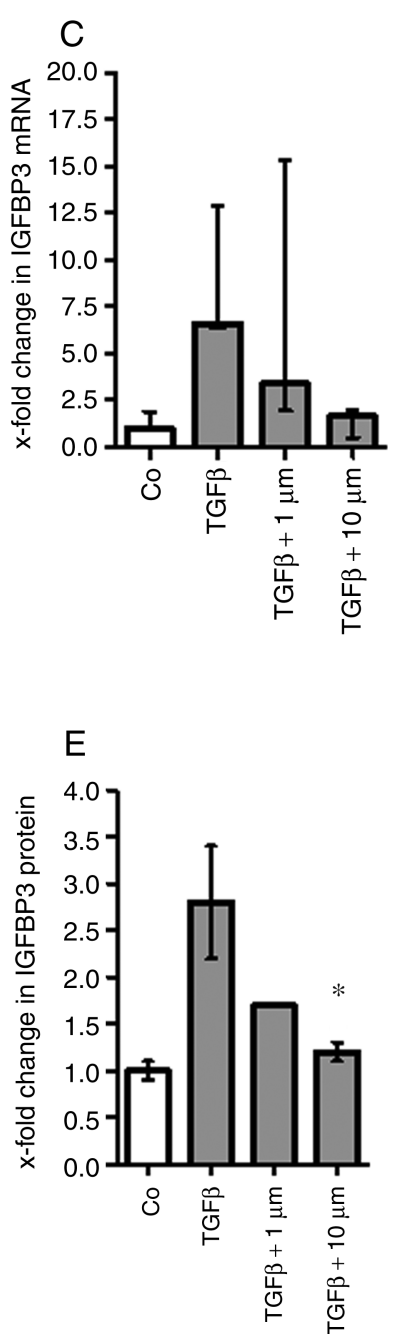

Figure 3 Soluble guanylate cyclase stimulation reverses transforming growth factor $\beta$ (TGF $\beta$ ) -induced fibroblast-to-myofibroblast differentiation of dermal fibroblasts. (A-E) Fibroblasts were differentiated with TGF $\beta$ for $72 \mathrm{~h}$ and subsequently incubated with $1.0 \mu \mathrm{M}$ or $10.0 \mu \mathrm{M}$ BAY $41-2272$ in the presence of $10 \mathrm{ng} / \mathrm{mL}$ TGF $\beta$ for additional $72 \mathrm{~h}$. All experiments are expressed as $x$-fold of the unstimulated control. $\mathrm{N}=3 \mathrm{for}$ all experiments. $\mathrm{A}$ paired, two-sided t test was used for statistical analysis. (A-C) ACTA2, CNN1 and IGFBP3 mRNA levels with HMBS as internal control. (D) Western blot analysis for $\alpha$-smooth muscle actin and calponin-1. $\alpha$-Tubulin served as loading control. Representative images from independent experiments using cells from three different donors are shown. (E) Secreted IGFBP3 protein levels as determined by ELISA.

that complex with SMAD4 to enter the nucleus, bind to specific DNA sequences and modulate gene transcription. Thus, we first investigated the expression of SMAD7, a classical TGF $\beta$ target genes. While stimulation of fibroblasts with TGF $\beta$ led to the expected increase of SMAD7 mRNA levels, pretreatment of fibroblasts with antifibrotic doses of BAY 41-2272 had no effects (figure 5A,B). Other SMAD-dependent TGF $\beta$ target genes, such as plasminogen activator inhibitor 1 (PAI1) and connective tissue growth factor (CTGF), showed similar responses (data not shown). Taking advantage of a 'SMAD-specific' CAGA reporter assay, we next showed that sGC stimulation did not modulate the transcription of classical TGF $\beta$ target sequences in fibroblasts (figure 5C). In western blot experiments and immunofluorescence stainings, we demonstrated that SMAD2 and 3 accumulated in the nucleus of fibroblasts upon stimulation with TGF $\beta$, which remained unchanged upon pretreatment with BAY 41-2272 (figure 5D,E). In addition, sGC stimulation did not affect the release of TGF $\beta$ from fibroblasts: BAY 41-2272 did not alter TGF $\beta$ mRNA expression in fibroblasts, and supernatants taken from fibroblasts treated with BAY 41-2272 did not reduce TGF $\beta$-dependent CAGA reporter activity when transferred to other fibroblast cultures (see online supplementary figure S1D,E). While these data suggested that sGC signalling does not interfere with canonical TGF $\beta$ signalling in dermal fibroblasts, additional analyses revealed that sGC stimulation reduced TGF $\beta$-dependent ERK phosphorylation without changing total ERK concentrations (figure 5F,G). Phosphorylation of JNK and Akt, representatives of other central non-canonical TGF $\beta$ pathways, remained unaffected (data not shown) by sGC stimulation.

\section{The sGC stimulator BAY 41-2272 is effective in a TGF $\beta$-driven murine model of dermal fibrosis}

To demonstrate that sGC signalling also interferes with the profibrotic activity of TGF $\beta$ signalling in vivo, we evaluated the effects of sGC stimulation in a mouse model of fibrosis induced by overexpression of a constitutively active TGF $\beta$ receptor I. In this model, treatment with the sGC stimulator BAY 41-2272 showed strong and dose-dependent antifibrotic effects: we observed a decrease in skin thickening by $51.9 \%$ (CI $38.4 \%$ to $63.8 \%$; figure $6 \mathrm{~A}, \mathrm{~B})$, in hydroxyproline content by $58.9 \%$ (CI $6.8 \%$ to $119.1 \%$; figure $6 \mathrm{C}$ ) and in the number of 

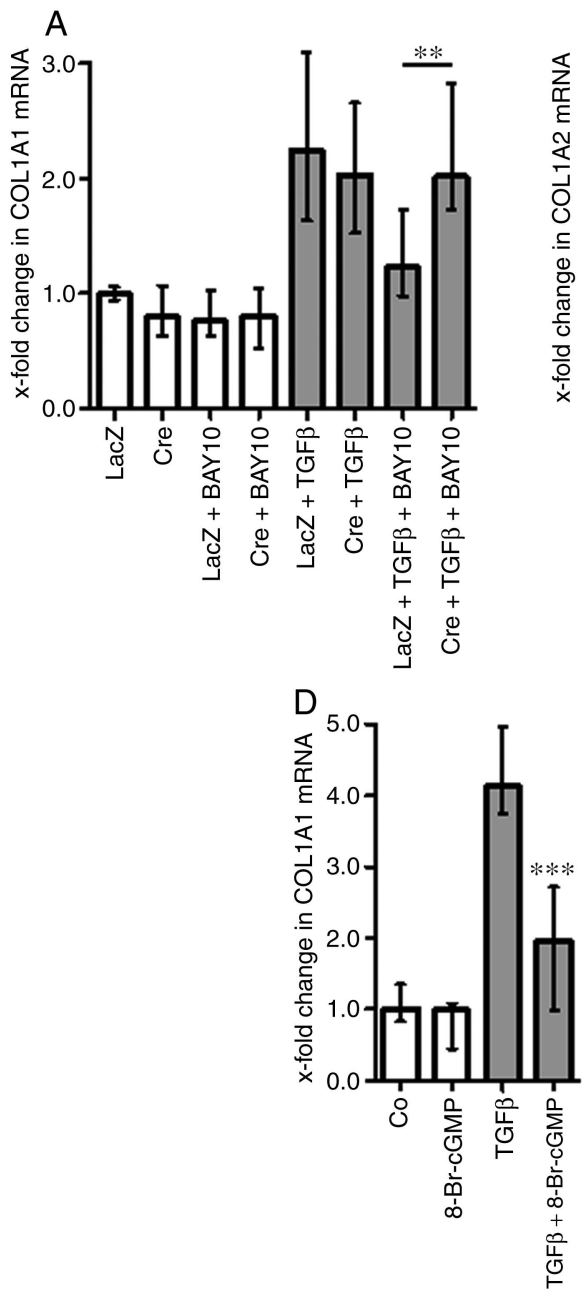
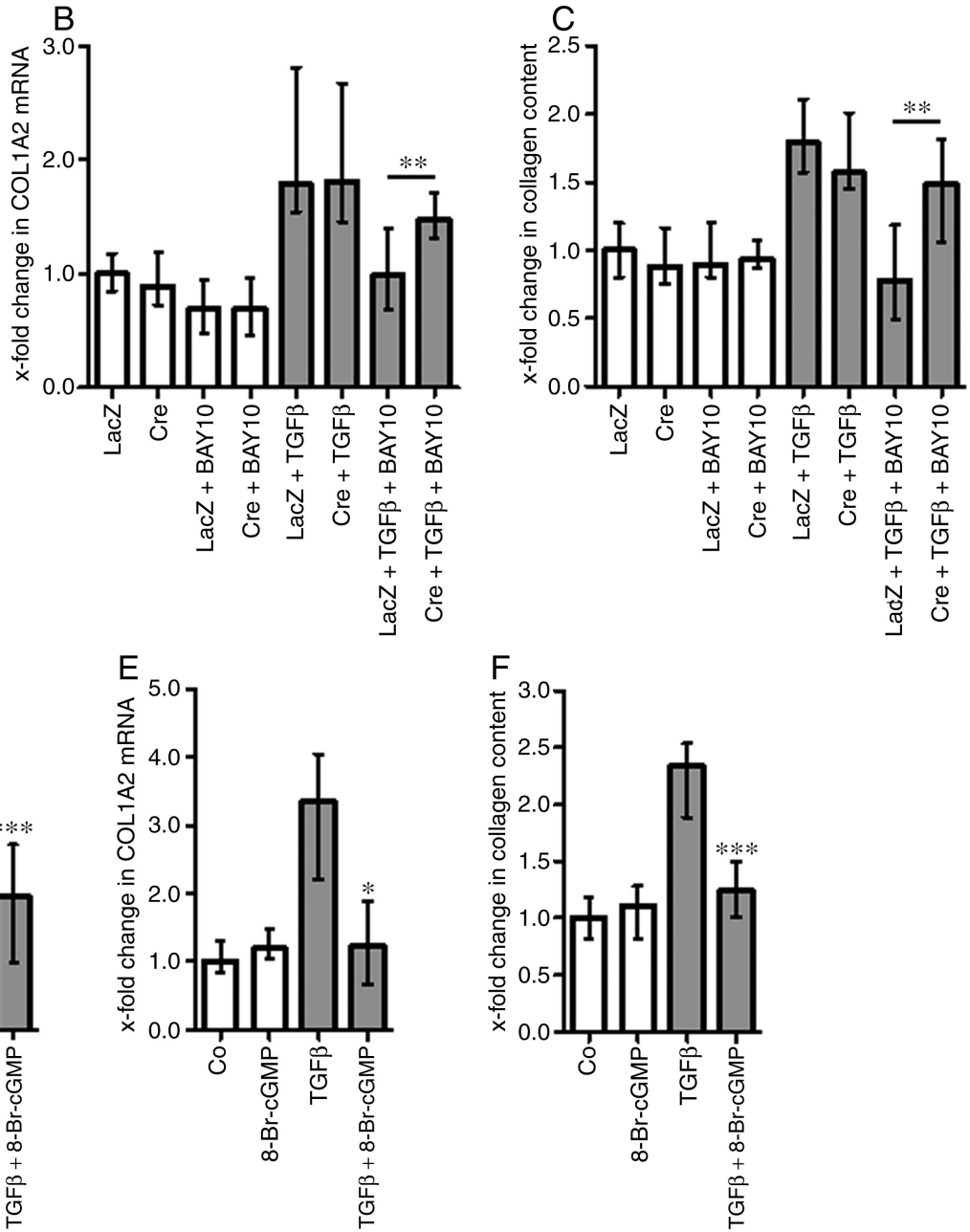

Figure 4 The antifibrotic effects of BAY 41-2272 depend on soluble guanylate cyclase (sGC) activity and are mediated via cyclic guanosine monophosphate (cGMP). (A-C) sGC-deficient fibroblasts were generated by isolation from $\mathrm{sGC}^{\mathrm{fl} / \mathrm{fl}}$ mice and recombination with Ad-Cre-GFP virus. Fibroblasts from $\mathrm{sGC}^{\mathrm{fl} / \mathrm{fl}}$ mice infected with Ad-LacZ virus served as controls. sGC-deficient and control fibroblasts were subjected to BAY $41-2272$ treatment and transforming growth factor $\beta$ (TGF $\beta$ ) stimulation (BAY $41-227210 \mu \mathrm{M}$; TGF $\beta 10 \mathrm{ng} / \mathrm{ml}$ ) for $48 \mathrm{~h}$. All experiments are expressed as $x$-fold of the unstimulated control. $\mathrm{N} \geq 6$ for all experiments. (A) COL1A1 mRNA levels with $\beta$-actin as internal control. (B) COL1A2 mRNA levels with $\beta$-actin as internal control. (C) Collagen content in the supernatant as measured by SirCol assay. (D-F) Human fibroblasts were incubated with $250 \mu \mathrm{M}$ 8-Bromo-cGMP, a stable analogue of the sGC product cGMP, and stimulated with TGF $\beta$ for $48 \mathrm{~h}$. All experiments are expressed as $x$-fold of the unstimulated control. $\mathrm{N} \geq 6$ for all experiments. (D) COL1A1 mRNA levels with $\beta$-actin as internal control. (E) COL1A2 mRNA levels with $\beta$-actin as internal control. (F) Collagen content in the supernatant as measured by SirCol assay.

aSMA-positive myofibroblasts by $59.8 \%$ (CI $46.9 \%$ to $126.7 \%$; figure 6D). In line with our in vitro findings, blockade of the pro-fibrotic TGF $\beta$ signalling was independent from nuclear accumulation of phosphorylated SMAD2 and 3 and expression of the TGF $\beta$ target gene SMAD7 (figure 6E,F). Together with our in vitro findings, these data highlight that sGC signalling interferes with the pro-fibrotic TGF $\beta$ signalling by blocking SMAD-independent pathways.

\section{DISCUSSION}

TGF $\beta$ signalling is one of the key signalling pathways in fibrosis. A large body of evidence demonstrates that enhanced TGF $\beta$ signalling drives fibroblast activation and collagen release in various fibrotic diseases, including SSc. ${ }^{1} 25^{2} 35$ In vitro studies highlight that TGF $\beta$ can induce a pro-fibrotic phenotype in resting fibroblasts, which closely reflects characteristics of fibroblasts isolated from lesional SSc skin. ${ }^{2} 36$ This phenotype is characterised by enhanced expression of contractile proteins (eg, $\alpha$-SMA) and stress fibres as well as by increased release of matrix proteins (eg, collagens). Given this central role in fibrosis, novel therapies that interfere with TGF $\beta$ signalling are considered promising therapeutic strategies in the treatment of fibrotic disease. ${ }^{4}$ Effective therapies to target TGF $\beta$ signalling in daily clinical practice, however, are still not available. ${ }^{4}$

We have recently demonstrated that stimulation of the $\mathrm{sGC}$ is effective in inhibiting inflammation-driven and inflammationindependent experimental dermal fibrosis. In our studies, the sGC stimulator BAY 41-2272 prevented and treated dermal fibrosis in the models of bleomycin-induced dermal fibrosis and in Tight skin-1 mice. Effective antifibrotic doses of the sGC stimulator were well tolerated and did not cause systemic hypotension as assessed by telemetry experiments. ${ }^{14}$ Since further studies demonstrated antifibrotic effects in preclinical models of liver $^{16}$ and kidney fibrosis, ${ }^{17} 18$ the sGC-cGMP axis could be a common modulator and therapeutic target of tissue remodelling and fibrosis of various organs.

Up to now, the antifibrotic mode of action of sGC-cGMP signalling has remained elusive. In the present study, we 

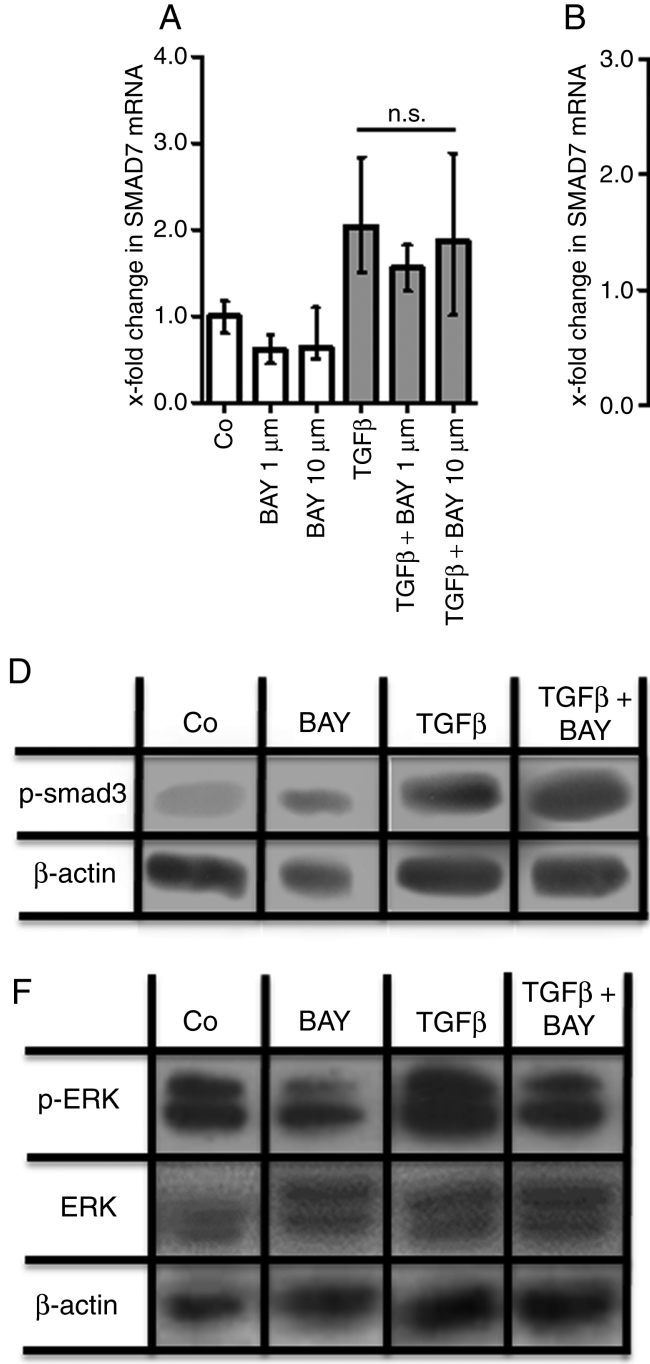
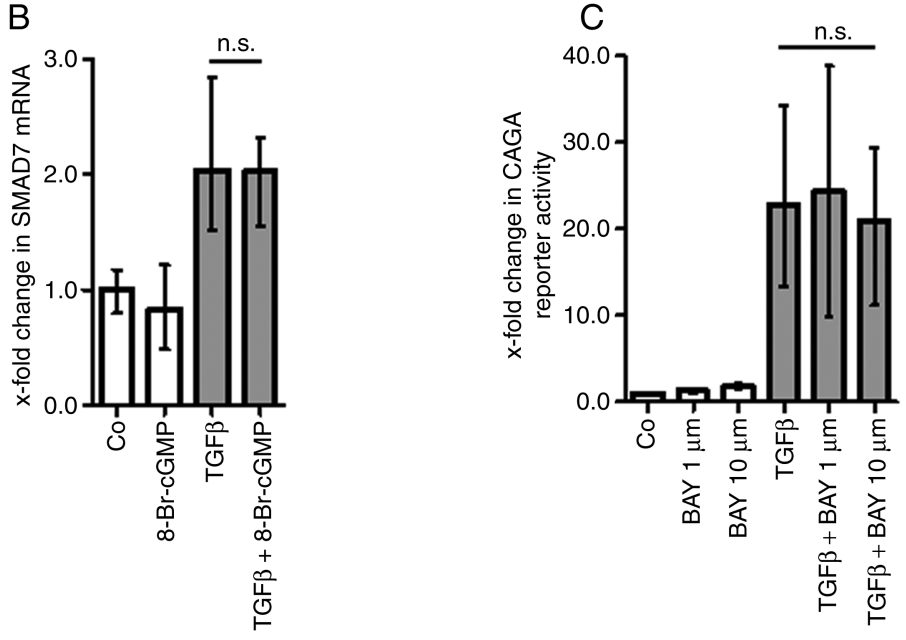

E

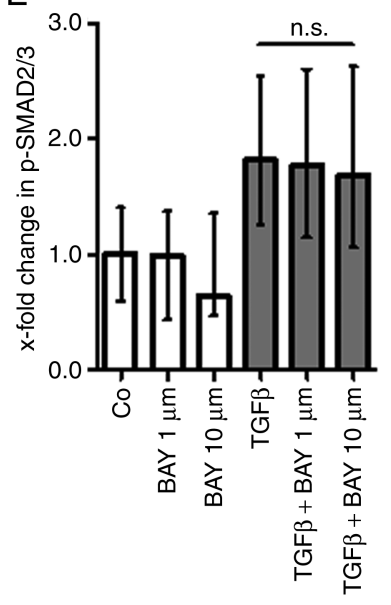

G

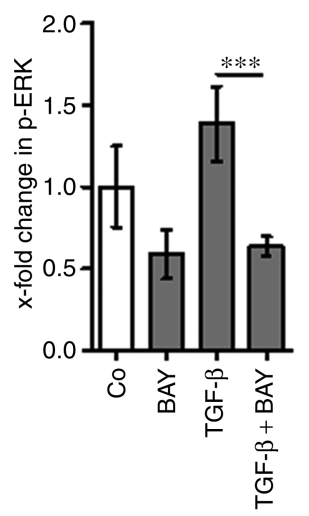

Figure 5 Soluble guanylate cyclase $(\mathrm{sGC})$ signalling is independent from canonical transforming growth factor $\beta$ (TGF $\beta$ ) signalling. (A-E) Normal human fibroblasts were treated with the sGC stimulator BAY 41-2272 (1.0 and 10.0 $\mu \mathrm{M}$ ) or the stable cyclic guanosine monophosphate (cGMP) analogue 8-Bromo-cGMP $(250 \mu \mathrm{M})$ and stimulated with TGF $\beta(10 \mathrm{ng} / \mathrm{mL})$. All experiments are expressed as $x$-fold of the unstimulated control. $N \geq 6$ for all experiments. (A, B) SMAD7 mRNA levels were analysed with $\beta$-actin as internal control after $48 \mathrm{~h}$ of TGF $\beta$ stimulation. (C) SMAD-dependent CAGA reporter activity was assessed by luciferase assay after $24 \mathrm{~h}$ of TGF $\beta$ stimulation. (D) Nuclear levels of SMAD3 upon treatment with $10.0 \mu \mathrm{M}$ BAY 41-2272 and $10 \mathrm{ng} / \mathrm{ml}$ TGF $\beta$ were assessed by western blot analysis after $3 \mathrm{~h}$ of TGF $\beta$ stimulation. (E) Levels of phosphorylated SMAD2 and 3 as assessed by immunofluorescence staining after $6 \mathrm{~h}$ of TGF $\beta$ stimulation. (F, G) Levels of ERK and phosphorylated ERK upon treatment with $10.0 \mu \mathrm{M}$ BAY 41-2272 and stimulation with $10 \mathrm{ng} / \mathrm{mL}$ TGF $\beta$ for $30 \mathrm{~min}$ were assessed by western blot analysis and quantified by Image J software.

investigated the molecular events mediating the antifibrotic effects of the sGC-cGMP axis and established a novel link between sGC and TGF $\beta$ signalling in fibrosis. We observed that pharmacological stimulation of $\mathrm{sGC}$ signalling inhibited TGF $\beta$-driven fibroblast activation and collagen release. Stimulation of the sGC could even reverse TGF $\beta$-driven fibroblast activation, which may be of utmost importance for therapeutic applications when fibrosis has already become manifest. By using knockout experiments, we confirmed that the sGC activity is essential for the antifibrotic effects of sGC stimulators. In our experiments with cultured dermal fibroblasts, sGC signalling did not inhibit the release of TGF $\beta$ as suggested for chronic heart remodelling and heart fibrosis. ${ }^{17} 37$ By contrast, sGC signalling interfered with non-canonical TGF $\beta$ cascades since the antifibrotic effects of the sGC were independent from SMAD2 and 3 signalling and TGF $\beta$ target gene transcription. Our data indicate that sGC activity interferes with TGF $\beta$-dependent ERK signalling, an observation that deserves more detailed investigations by future studies. Of note, the novel antifibrotic crosstalk between sGC and non-canonical TGF $\beta$ signalling is also active in vivo. In experimental fibrosis induced by overexpression of a constitutively active TGF- $\beta 1$ receptor, sGC stimulation by BAY 41-2272 inhibited SMAD-independent TGF $\beta$ signalling and reduced dermal fibrosis. Thus, sGC stimulators represent auspicious candidates to target TGF $\beta$ signalling and treat fibrosis in human disease.

Recently, phase 3 clinical trials have shown excellent efficacy and tolerability of the sGC stimulator riociguat in $\mathrm{PAH}$ and CTPH. ${ }^{12}{ }^{13}$ BAY 41-2272, which we used in our fibrosis models, is the lead compound for riociguat and therefore shows a close chemical and biological relationship. In the PAH trial, around one quarter of patients receiving riociguat suffered from an underlying connective tissue disease and, presumably, most of them from SSc. ${ }^{12}$ Detailed subgroup analyses including information about fibrotic disease manifestations in the SSc-PAH patients are unfortunately not available (personal communication). Nevertheless, based on our preclinical findings, it is tempting to speculate that sGC stimulation might be beneficial for both PAH 
A AdLacZ + vehicle adTBR + vehicle

adTBR + BAY $1 \mathrm{mg} / \mathrm{kg}$ bid
$\operatorname{adTBR}+$ BAY $3 \mathrm{mg} / \mathrm{kg}$ bid
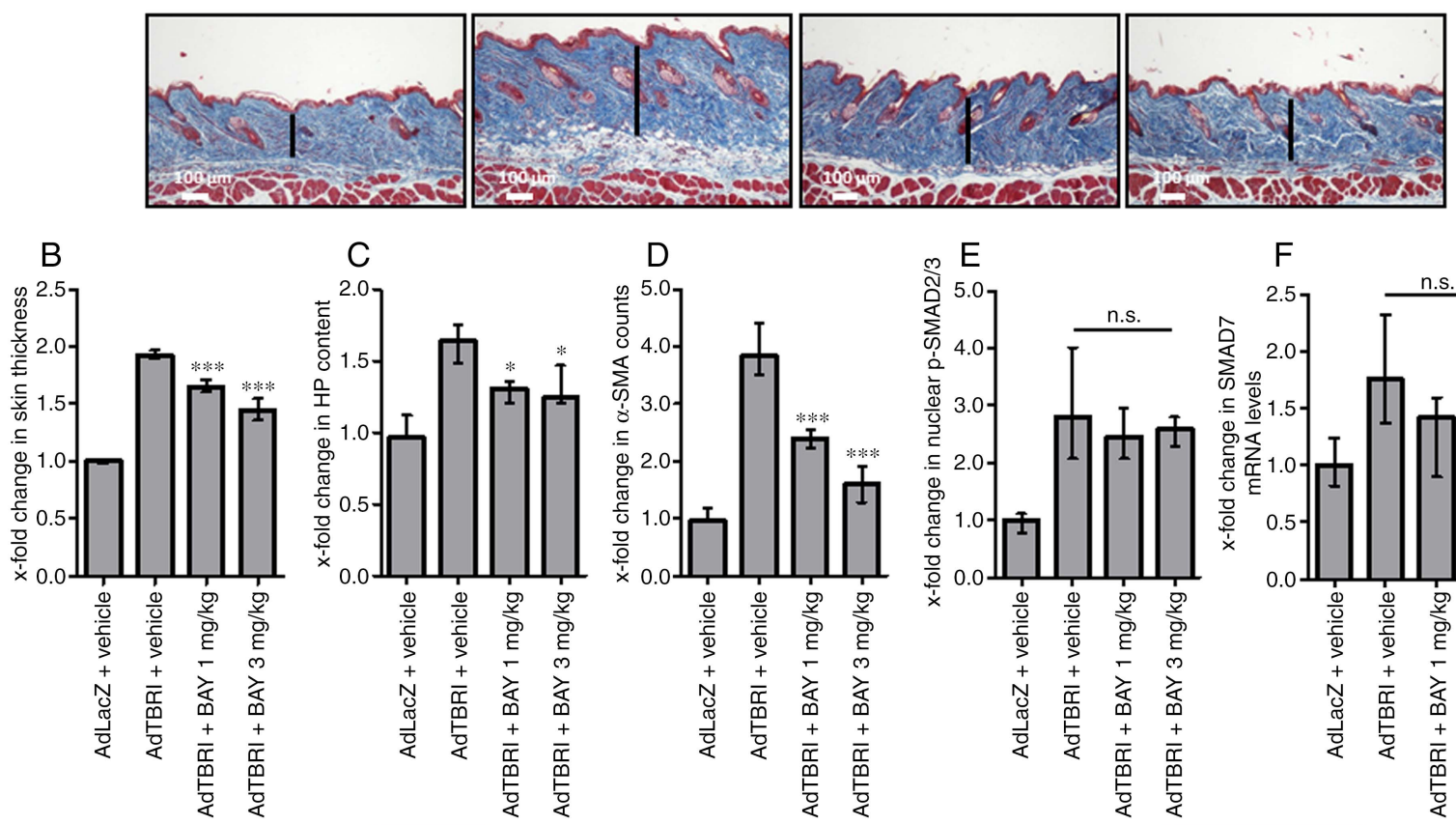

$\mathrm{F}$ fibrosis

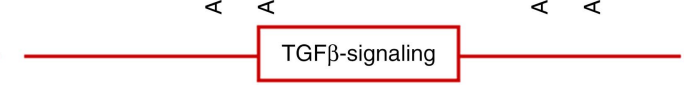

Figure 6 Soluble guanylate cyclase stimulation by BAY 41-2272 inhibits dermal fibrosis in mice challenged with an adenovirus encoding for a constitutively active transforming growth factor $\beta$ (TGF $\beta$ ) receptor 1 (adTBRI). (A-F) All experiments are expressed as $x$-fold of the unstimulated control. $\mathrm{N}=8$ for all experiments. (A) Representative Masson's trichrome stains with collagens staining in blue. (B) Dermal thickness as assessed on Masson's trichrome stains. Scale bar $100 \mu \mathrm{m}$. (C) Dermal hydroxyproline content. (D) Numbers of dermal $\alpha$-smooth muscle actin-positive fibroblasts. (E) Numbers of dermal fibroblasts showing extensive nuclear staining of phosphorylated SMAD2 and 3. (F) Messenger RNA levels of the TGF $\beta$ target gene SMAD7 as assessed by quantitative real-time PCR.

and fibrosis in SSc. In addition to PAH as large vessel complication, almost all patients with SSc suffer from small vessel vasculopathy. Given the mode of action of sGC stimulators, activation of the sGC-cGMP signalling might also improve small vessel disease in SSc. These speculations, however, still need thorough preclinical and clinical testing.

sGC stimulators might represent a novel class of drugs to simultaneously target fibrosis and vascular disease in patients with SSc. Preclinical models of SSc that reflect both vascular and fibrotic disease, such as the Fra-2 transgenic mice or the UCD-200 chicken, may help to further corroborate this hypothesis. Apart from beneficial effects in dermal fibrosis and SSc, sGC stimulators might effectively treat other fibrotic diseases as suggested from preclinical studies. ${ }^{14}{ }^{16-18}$ Future studies, however, still need to address whether similar molecular mechanisms mediate the antifibrotic effects of cGMP in other fibrotic conditions.

Taken together, we have established a novel link between the antifibrotic sGC signalling and the pro-fibrotic TGF $\beta$ pathway. Stimulation of the sGC inhibits experimental fibrosis by interfering with the SMAD-independent ERK cascades. Stimulators of the sGC have already completed late clinical evaluation for PAH and CTPH and could therefore be readily applied in clinical studies with patients suffering from SSc and other fibrotic diseases.

\section{Author affiliations}

${ }^{1}$ Department of Internal Medicine 3 and Institute for Clinical Immunology, University of Erlangen-Nuremberg, Erlangen, Germany

${ }^{2}$ Institute for Biomedical Aging Research, University of Innsbruck, Innsbruck, Austria
${ }^{3}$ Department of Internal Medicine, Innsbruck Medical University, Innsbruck, Austria ${ }^{4}$ Department of Trauma and Orthopaedic Surgery, University Erlangen-Nuremberg, Erlangen, Germany

${ }^{5}$ Institute for Physiology, Julius-Maximilians-University Würzburg, Würzburg, Germany

${ }^{6}$ Bayer Health Care, Global Drug Discovery—Common Mechanism Research, Wuppertal, Germany

${ }^{7}$ Hannover Medical School, Institute of Pharmacology, Hannover, Germany ${ }^{8}$ Department of Rheumatology, University Hospital Zurich, Zürich, Switzerland

Acknowledgements We thank Regina Kleinlein, Corinna Mohr, Katja Dreißigacker, Verena Wäsch and Martin Heitz for excellent technical assistance.

Contributors $C B, J H W D, P S$ and $O D$ were responsible for study design. $C B, C Z$, $K P-Z, R M, A D, C D, J H, C M, H J$ and MP were responsible for data acquisition. $C B$, $J H W D, O D, G S, A F, P B$ and $P S$ were responsible for interpretation of results. $C B$, $J H W D, C Z$ and PB were responsible for the overall content of the manuscript.

Funding Grant support was provided by the Erlanger Leistungsbezogene Anschubfinanzierung und Nachwuchsföderung (ELAN), grants J29 and A57 of the Interdisciplinary Center of Clinical Research (IZKF) in Erlangen and grants DI 1537/ 1-1, DI 1537/2-1, DI 1537/4-1, AK 144/1-1, BE 5191/1-1, SCHE 1583/7-1, and FR 1725/1-5 from the Deutsche Forschungsgemeinschaft. In addition, the study was supported by the Career Support Award of Medicine of the Ernst Jung Foundation (to JHWD).

Competing interests $\mathrm{OD}$ has consultancy relationships and/or has received research funding from Actelion, Pfizer, Ergonex, BMS, Sanofi-Aventis, United BioSource Corporation, Roche/Genentech, medac, Biovitrium, Boehringer Ingelheim Pharma, Novartis, 4 D Science, Active Biotec, Bayer-Schering, Sinoxa, Serodapharm and EpiPharm. JHWD has consultancy relationships and/or has received research funding from Actelion, Pfizer, Ergonex, BMS, Celgene, Bayer Pharma, JB Therapeutics, Sanofi-Aventis, Novartis, Array Biopharma and Active Biotec in the area of potential treatments of $S S C$ and is stock owner of $4 D$ Science. PS is employee of Bayer Health Care. CZ and PB have consultancy relationship to Bayer Pharma.

Ethics approval University of Erlangen-Nuremberg. 
Provenance and peer review Not commissioned; externally peer reviewed.

\section{REFERENCES}

1 Gabrielli A, Avvedimento EV, Krieg T. Scleroderma. N Engl J Med 2009;360:1989-2003.

2 Varga J, Abraham D. Systemic sclerosis: a prototypic multisystem fibrotic disorder. J Clin Invest 2007;117:557-67.

3 Beyer C, Distler JH. Tyrosine kinase signaling in fibrotic disorders: Translation of basic research to human disease. Biochim Biophys Acta 2013;1832:897-904.

4 Beyer C, Distler O, Distler JH. Innovative antifibrotic therapies in systemic sclerosis. Curr Opin Rheumatol 2012;24:274-80.

5 Wynn TA, Ramalingam TR. Mechanisms of fibrosis: therapeutic translation for fibrotic disease. Nat Med 2012;18:1028-40.

6 Kramer M, Dees C, Huang J, et al. Inhibition of H3K27 histone trimethylation activates fibroblasts and induces fibrosis. Ann Rheum Dis 2013;72:614-20.

7 Khodzhigorova A, Distler A, Lang V, et al. Inhibition of sumoylation prevents experimental fibrosis. Ann Rheum Dis 2012;71:1904-8.

8 Huber LC, Distler JH, Moritz F, et al. Trichostatin A prevents the accumulation of extracellular matrix in a mouse model of bleomycin-induced skin fibrosis. Arthritis Rheum 2007;56:2755-64.

9 Bechtel W, McGoohan S, Zeisberg EM, et al. Methylation determines fibroblast activation and fibrogenesis in the kidney. Nat Med 2010;16:544-50.

10 Evgenov OV, Pacher P, Schmidt PM, et al. NO-independent stimulators and activators of soluble guanylate cyclase: discovery and therapeutic potential. Nat Rev Drug Discov 2006;5:755-68.

11 Megson IL, Miller MR. NO and sGC-stimulating NO donors. Handb Exp Pharmacol 2009:247-76.

12 Ghofrani HA, Galie N, Grimminger F, et al. Riociguat for the treatment of pulmonary arterial hypertension. N Engl J Med 2013;369:330-40.

13 Ghofrani HA, D'Armini AM, Grimminger F, et al. Riociguat for the treatment of chronic thromboembolic pulmonary hypertension. N Engl J Med 2013;369: 319-29.

14 Beyer C, Reich N, Schindler SC, et al. Stimulation of soluble guanylate cyclase reduces experimental dermal fibrosis. Ann Rheum Dis 2012;71:1019-26.

15 Sampson N, Berger P, Zenzmaier C. Therapeutic targeting of redox signaling in myofibroblast differentiation and age-related fibrotic disease. Oxid Med Cell Longev 2012;2012:458276.

16 Knorr A, Hirth-Dietrich C, Alonso-Alija C, et al. Nitric oxide-independent activation of soluble guanylate cyclase by BAY 60-2770 in experimental liver fibrosis. Arzneimittelforschung 2008:58:71-80.

17 Geschka S, Kretschmer A, Sharkovska Y, et al. Soluble guanylate cyclase stimulation prevents fibrotic tissue remodeling and improves survival in salt-sensitive dahl rats. PLOS ONE 2011;6:e21853.

18 Wang Y, Kramer S, Loof T, et al. Enhancing CGMP in experimental progressive renal fibrosis: soluble guanylate cyclase stimulation vs. phosphodiesterase inhibition. Am J Physiol Renal Physiol 2006;290:F167-76.

19 Dees C, Akhmetshina A, Zerr $\mathrm{P}$, et al. Platelet-derived serotonin links vascular disease and tissue fibrosis. J Exp Med 2011;208:961-72.
20 Akhmetshina A, Palumbo K, Dees C, et al. Activation of canonical Wnt signalling is required for TGF-beta-mediated fibrosis. Nat Commun 2012;3:735.

21 Distler JH, Distler O. Imatinib as a novel therapeutic approach for fibrotic disorders. Rheumatology (Oxford) 2009;48:2-4.

22 Mergia E, Koesling D, Friebe A. Genetic mouse models of the NO receptor 'soluble' guanylyl cyclases. Handb Exp Pharmacol 2009;191:33-46.

23 Friebe A, Mergia E, Dangel O, et al. Fatal gastrointestinal obstruction and hypertension in mice lacking nitric oxide-sensitive guanylyl cyclase. Proc Natl Acad Sci USA 2007;104:7699-704

24 Beyer C, Schramm A, Akhmetshina A, et al. beta-catenin is a central mediator of pro-fibrotic Wnt signaling in systemic sclerosis. Ann Rheum Dis 2012;71:761-7.

25 Reich N, Tomcik M, Zerr P, et al. Jun N-terminal kinase as a potential molecular target for prevention and treatment of dermal fibrosis. Ann Rheum Dis 2012;71:737-45

26 Zerr $\mathrm{P}$, Vollath $\mathrm{S}$, Palumbo-Zerr $\mathrm{K}$, et al. Vitamin $\mathrm{D}$ receptor regulates TGF-beta signalling in systemic sclerosis. Ann Rheum Dis 2015;74:e20.

27 Palumbo K, Zerr $\mathrm{P}$, Tomcik M, et al. The transcription factor JunD mediates transforming growth factor $\{$ beta\}-induced fibroblast activation and fibrosis in systemic sclerosis. Ann Rheum Dis 2011;70:1320-6.

28 Avouac J, Palumbo K, Tomcik M, et al. Inhibition of activator protein 1 signaling abrogates transforming growth factor beta-mediated activation of fibroblasts and prevents experimental fibrosis. Arthritis Rheum 2012;64:1642-52.

29 Beyer $\mathrm{C}$, Reichert $\mathrm{H}$, Akan $\mathrm{H}$, et al. Blockade of canonical Wnt signalling ameliorates experimental dermal fibrosis. Ann Rheum Dis 2013;72:1255-8.

30 Beyer C, Skapenko A, Distler A, et al. Activation of pregnane $X$ receptor inhibits experimental dermal fibrosis. Ann Rheum Dis 2013;72:621-5.

31 Distler A, Lang V, Del Vecchio T, et al. Combined inhibition of morphogen pathways demonstrates additive antifibrotic effects and improved tolerability. Ann Rheum Dis 2014;73:1264-8.

32 Zhang Y, Dees C, Beyer C, et al. Inhibition of casein kinase II reduces TGFbeta induced fibroblast activation and ameliorates experimental fibrosis. Ann Rheum Dis 2015;74:936-43.

33 Zenzmaier C, Kern J, Sampson N, et al. Phosphodiesterase Type 5 Inhibition Reverts Prostate Fibroblast-to-Myofibroblast Trans-Differentiation. Endocrinology 2012;153:5546-55.

34 Zenzmaier C, Sampson N, Pernkopf D, et al. Attenuated proliferation and trans-differentiation of prostatic stromal cells indicate suitability of phosphodiesterase type 5 inhibitors for prevention and treatment of benign prostatic hyperplasia. Endocrinology 2010;151:3975-84.

35 Varga J, Pasche B. Transforming growth factor beta as a therapeutic target in systemic sclerosis. Nat Rev Rheumatol 2009;5:200-6.

36 Pannu J, Trojanowska M. Recent advances in fibroblast signaling and biology in scleroderma. Curr Opin Rheumatol 2004;16:739-45.

37 Irvine JC, Ganthavee V, Love JE, et al. The soluble guanylyl cyclase activator bay 58-2667 selectively limits cardiomyocyte hypertrophy. PLOS ONE 2012; 7:e44481.

38 Masuyama H, Tsuruda T, Sekita Y, et al. Pressure-independent effects of pharmacological stimulation of soluble guanylate cyclase on fibrosis in pressure-overloaded rat heart. Hypertens Res 2009;32:597-603. 


\section{ARD Stimulation of the soluble guanylate cyclase (sGC) inhibits fibrosis by blocking non-canonical TGF $\beta$ signalling}

Christian Beyer, Christoph Zenzmaier, Katrin Palumbo-Zerr, Rossella Mancuso, Alfiya Distler, Clara Dees, Pawel Zerr, Jingang Huang, Christiane Maier, Milena L Pachowsky, Andreas Friebe, Peter Sandner, Oliver Distler, Georg Schett, Peter Berger and Jörg H W Distler

Ann Rheum Dis 2015 74: 1408-1416 originally published online February 23, 2014

doi: 10.1136/annrheumdis-2013-204508

Updated information and services can be found at:

http://ard.bmj.com/content/74/7/1408

These include: Supplementary
Material

References

Email alerting service
Supplementary material can be found at: http://ard.bmj.com/content/suppl/2014/02/24/annrheumdis-2013-2045 08.DC1

This article cites 36 articles, 13 of which you can access for free at: http://ard.bmj.com/content/74/7/1408\#BIBL

Receive free email alerts when new articles cite this article. Sign up in the box at the top right corner of the online article.

Topic Articles on similar topics can be found in the following collections Collections

\section{Notes}

To request permissions go to:

http://group.bmj.com/group/rights-licensing/permissions

To order reprints go to:

http://journals.bmj.com/cgi/reprintform

To subscribe to BMJ go to:

http://group.bmj.com/subscribe/ 